\title{
Intrinsic Ultracontractivity for Domains in Negatively Curved Manifolds
}

\author{
Hiroaki Aikawa ${ }^{1} \cdot$ Michiel van den Berg ${ }^{2} \cdot$ Jun Masamune ${ }^{3}$ \\ Received: 21 December 2020 / Revised: 21 March 2021 / Accepted: 21 April 2021 / \\ Published online: 8 September 2021 \\ () The Author(s) 2021
}

\begin{abstract}
Let $M$ be a complete, non-compact, connected Riemannian manifold with Ricci curvature bounded from below by a negative constant. A sufficient condition is obtained for open and connected sets $D$ in $M$ for which the corresponding Dirichlet heat semigroup is intrinsically ultracontractive. That condition is formulated in terms of capacitary width. It is shown that both the reciprocal of the bottom of the spectrum of the Dirichlet Laplacian acting in $L^{2}(D)$, and the supremum of the torsion function for $D$ are comparable with the square of the capacitary width for $D$ if the latter is sufficiently small. The technical key ingredients are the volume doubling property, the Poincaré inequality and the Li-Yau Gaussian estimate for the Dirichlet heat kernel at finite scale.
\end{abstract}

Keywords Intrinsic ultracontractivity $\cdot$ Ricci curvature $\cdot$ First eigenvalue $\cdot$ Heat kernel · Torsion function · Capacitary width

Dedicated to the memory of Professor Walter K. Hayman.

Communicated by Tom Carroll.

This work was supported by JSPS KAKENHI Grant Number 17H01092. MvdB was also supported by The Leverhulme Trust through Emeritus Fellowship EM-2018-011-9.

\iroaki Aikawa

aikawa@isc.chubu.ac.jp

Michiel van den Berg

mamvdb@bristol.ac.uk

Jun Masamune

jmasamune@math.sci.hokudai.ac.jp

1 College of Engineering, Chubu University, Kasugai 487-8501, Japan

2 School of Mathematics, University of Bristol, Fry Building, Woodland Road, Bristol BS8 1UG, UK

3 Department of Mathematics, Hokkaido University, Sapporo 060-0810, Japan 
Mathematics Subject Classification $31 \mathrm{C} 12 \cdot 31 \mathrm{~B} 15 \cdot 58 \mathrm{~J} 35$

\section{Main Results}

Let $M$ be a complete, non-compact, $n$-dimensional connected Riemannian manifold, without boundary, and with Ricci curvature bounded below by a negative constant, i.e., Ric $\geq-K$ with non-negative constant $K$. Throughout the paper, $K$ is reserved for this constant. In this article, we investigate domains (open, and connected sets) in $M$ for which the heat semigroup is intrinsically ultracontractive.

For a domain $D \subset M$ we denote by $p_{D}(t, x, y), t>0, x, y \in D$, the Dirichlet heat kernel for $\partial / \partial t-\Delta$ in $D$, i.e., the fundamental solution to $(\partial / \partial t-\Delta) u=0$ subject to the Dirichlet boundary condition $u(t, x)=0$ for $x \in \partial D$ and $t>0$. Davies and Simon [12] introduced the notion of intrinsic ultracontractivity. There are several equivalent definitions for intrinsic ultracontractivity ( [12, p. 345]). The following is in terms of the heat kernel estimate.

Definition 1.1 Let $D \subset M$. We say that the semigroup associated with $p_{D}(t, x, y)$ is intrinsically ultracontractive (abbreviated to IU) if the following two conditions are satisfied:

(i) The Dirichlet Laplacian $-\Delta$ has no essential spectrum and has the first eigenvalue $\lambda_{D}>0$ with corresponding positive eigenfunction $\varphi_{D}$ normalized by $\left\|\varphi_{D}\right\|_{2}=1$.

(ii) For every $t>0$, there exist constants $0<c_{t}<C_{t}$ depending on $t$ such that

$$
c_{t} \varphi_{D}(x) \varphi_{D}(y) \leq p_{D}(t, x, y) \leq C_{t} \varphi_{D}(x) \varphi_{D}(y) \text { for all } x, y \in D .
$$

For simplicity, we say that $D$ itself is IU if the semigroup associated with $p_{D}(t, x, y)$ is IU.

Both the analytic and probabilistic aspects of IU have been investigated in detail. For example it turns out that IU implies the Cranston-McConnell inequality, while IU is derived from very weak regularity of the domain. Davis [13] showed that a bounded Euclidean domain above the graph of an upper semi-continuous function is IU; no regularity of the boundary function is needed. There are many results on IU for Euclidean domains. Bañuelos and Davis [5, Thm. 1, Thm. 2] gave conditions characterizing IU and the Cranston-McConnell inequality when restricting to a certain class of plane domains, which illustrate subtle differences between IU and the Cranston-McConnell inequality. Méndez-Hernández [16] gave further extensions. See also [1,4,7,8,13], and references therein.

There are relatively few results for domains in a Riemannian manifold. Lierl and Saloff-Coste [15] studied a general framework including Riemannian manifolds. In that paper, they gave a precise heat kernel estimate for a bounded inner uniform domain, which implies IU ([15, Thm. 7.9]). In view of [13], however, the requirement of inner uniformity for IU to hold can be relaxed. See Sect. 7.

Our main result is a sufficient condition for IU for domains in a manifold, which is a generalization of the Euclidean case [1]. Our condition is given in terms of capacity. 
It is applicable not only to bounded domains but also to unbounded domains. Let $\Omega \subset M$ be an open set. For $E \subset \Omega$ we define relative capacity by

$$
\operatorname{Cap}_{\Omega}(E)=\inf \left\{\int_{\Omega}|\nabla \varphi|^{2} d \mu: \varphi \geq 1 \text { on } E, \varphi \in C_{0}^{\infty}(\Omega)\right\},
$$

where $\mu$ is the Riemannian measure in $M$ and $C_{0}^{\infty}(\Omega)$ is the space of all smooth functions compactly supported in $\Omega$. Let $d(x, y)$ be the distance between $x$ and $y$ in $M$. The open geodesic ball with center $x$ and radius $r>0$ is denoted by $B(x, r)=$ $\{y \in M: d(x, y)<r\}$. The closure of a set $E$ is denoted by $\bar{E}$, and so $\bar{B}(x, r)$ stands for the closed geodesic ball of center $x$ and radius $r$.

Definition 1.2 Let $0<\eta<1$. For an open set $D$ we define the capacitary width $w_{\eta}(D)$ by

$$
w_{\eta}(D)=\inf \left\{r>0: \frac{\operatorname{Cap}_{B(x, 2 r)}(\bar{B}(x, r) \backslash D)}{\operatorname{Cap}_{B(x, 2 r)}(\bar{B}(x, r))} \geq \eta \text { for all } x \in D\right\} .
$$

The next theorem asserts that the parameter $\eta$ has no significance.

Theorem 1.3 Let $0<R_{0}<\infty$. If $0<\eta^{\prime}<\eta<1$, then

$$
w_{\eta^{\prime}}(D) \leq w_{\eta}(D) \leq C w_{\eta^{\prime}}(D) \text { for all open sets } D \text { with } w_{\eta}(D)<R_{0}
$$

with $C>1$ depending only on $\eta, \eta^{\prime}, \sqrt{K} R_{0}$ and $n$.

The first condition for IU has a characterization in terms of capacitary width. This is straightforward from Persson's argument [17], and Theorem 1.6 below. Hereafter we fix $o \in M$.

Theorem 1.4 Let $D$ be a domain in $M$. Then $D$ has no essential spectrum if and only if $\lim _{R \rightarrow \infty} w_{\eta}(D \backslash \bar{B}(o, R))=0$.

We shall prove the following sufficient condition for IU, which looks the same as in the Euclidean case [1]. Nevertheless, the proof is significantly different for negatively curved manifolds. See the remark after Theorem A.

Theorem 1.5 Suppose $M$ has positive injectivity radius. Then a domain $D \subset M$ is IU if the following two conditions are satisfied:

(i) $\lim _{R \rightarrow \infty} w_{\eta}(D \backslash \bar{B}(o, R))=0$.

(ii) For some $\tau>0$

$$
\int_{0}^{\tau} w_{\eta}\left(\left\{x \in D: G_{D}(x, o)<t\right\}\right)^{2} \frac{d t}{t}<\infty
$$

where $G_{D}$ is the Green function for $D$. 
Our results are based on the relationship between the torsion function

$$
v_{D}(x)=\int_{D} G_{D}(x, y) d \mu(y)
$$

and the bottom of the spectrum

$$
\lambda_{\min }(D)=\inf \left\{\frac{\|\nabla f\|_{2}^{2}}{\|f\|_{2}^{2}}: f \in C_{0}^{\infty}(D) \text { with }\|f\|_{2} \neq 0\right\} .
$$

We note that $\lambda_{\min }(D)$ is the first eigenvalue $\lambda_{D}$ if $D$ has no essential spectrum. This is always the case for a bounded domain $D$. Theorem 1.4 asserts that the same holds even for an unbounded domain $D$ whenever $\lim _{R \rightarrow \infty} w_{\eta}(D \backslash \bar{B}(o, R))=0$. We also observe that the torsion function is the solution to the de Saint-Venant problem:

$$
\begin{aligned}
-\Delta v_{D}=1 & \text { in } D, \\
v_{D}=0 & \text { on } \partial D,
\end{aligned}
$$

where the boundary condition is taken in the Sobolev sense. The second named author [19] proved the following theorem.

Theorem A Let $K=0$. If $D \subset M$ satisfies $\lambda_{\min }(D)>0$, then

$$
\lambda_{\min }(D)^{-1} \leq\left\|v_{D}\right\|_{\infty} \leq C \lambda_{\min }(D)^{-1},
$$

where $C$ depends only on $M$.

The second inequality of (1.4) does not necessarily hold for negatively curved manifolds. Let $\mathbb{H}^{n}$ be the $n$-dimensional hyperbolic space of constant curvature -1 . It is known that

$$
\lambda_{\min }\left(\mathbb{H}^{n}\right)=\frac{(n-1)^{2}}{4},
$$

whereas $v_{\mathbb{H} n^{n}} \equiv \infty$ as $\mathbb{H}^{n}$ is stochastically complete. Hence the second inequality of (1.4) fails to hold if $D$ is the whole space $\mathbb{H}^{n}$.

The point of this paper is that (1.4) still holds if $D$ is limited to a certain class. We make use of (1.4) with this limitation to derive Theorems 1.4 and 1.5. We have the following theorem, which is a key ingredient in their proofs.

Theorem 1.6 Let $K \geq 0$ and let $0<\eta<1$. Then there exist $R_{0}>0$ and $C>1$ depending only on $K, \eta$ and $n$ such that if $D \subset M$ satisfies $w_{\eta}(D)<R_{0}$, then

$$
\frac{C^{-1}}{w_{\eta}(D)^{2}} \leq \frac{1}{\left\|v_{D}\right\|_{\infty}} \leq \lambda_{\min }(D) \leq \frac{C}{\left\|v_{D}\right\|_{\infty}} \leq \frac{C^{2}}{w_{\eta}(D)^{2}}
$$


Remark 1.7 We actually find $\Lambda_{0}>0$ depending only on $K$ and $n$ such that (1.4) holds for $D$ with $\lambda_{\min }(D)>\Lambda_{0}$ (Lemma 3.2 below). This is a generalization of Theorem A as $\Lambda_{0}=0$ for $K=0$. In practice, however, the condition $w_{\eta}(D)<R_{0}$ in Theorem 1.6 is more convenient since the capacitary width $w_{\eta}(D)$ can be more easily estimated than the bottom of the spectrum $\lambda_{\min }(D)$.

In Sect. 2 we summarize the key technical ingredients of the proofs: the volume doubling property, the Poincaré inequality and the Li-Yau Gaussian estimate for the Dirichlet heat kernel at finite scale. Observe that these fundamental tools are available not only for manifolds with Ricci curvature bounded below by a negative constant but also for unimodular Lie groups and homogeneous spaces. See [15, Ex. 2.11] and [18, Sect. 5.6]. This observation suggests that our approach is also extendable to those spaces.

We use the following notation. By the symbol $C$ we denote an absolute positive constant whose value is unimportant and may change from one occurrence to the next. If necessary, we use $C_{0}, C_{1}, \ldots$, to specify them. We say that $f$ and $g$ are comparable and write $f \approx g$ if two positive quantities $f$ and $g$ satisfy $C^{-1} \leq f / g \leq C$ with some constant $C \geq 1$. The constant $C$ is referred to as the constant of comparison.

\section{Preliminaries}

We recall that $M$ is a manifold of dimension $n \geq 2$ with Ric $\geq-K$ with $K \geq 0$. Let us recall the volume doubling property of the Riemannian measure $\mu$, the Poincare inequality and the Gaussian estimate for the Dirichlet heat kernel $p_{M}(t, x, y)$ for $M$. For $B=B(x, r)$ and $\tau>0$ we write $\tau B=B(x, \tau r)$.

Theorem 2.1 (Volume doubling at finite scale. [18, Thm. 5.6.4]) Let $0<R_{0}<\infty$. Then for all $B=B(x, r)$ with $0<r<R_{0}$

$$
\mu(2 B) \leq 2^{n} \exp \left(\sqrt{(n-1) K} R_{0}\right) \mu(B) .
$$

Theorem 2.2 (Poincaré inequality [18, Thm. 5.6.6]) For each $1 \leq p<\infty$ there exist positive constants $C_{n, p}$ and $C_{n}$ such that

$$
\int_{B}\left|f-f_{B}\right|^{p} d \mu \leq C_{n, p} r^{p} \exp \left(C_{n} \sqrt{K} r\right) \int_{2 B}|\nabla f|^{p} d \mu
$$

for all $B=B(x, r)$. Here $f_{B}$ stands for the average of $f$ on $B$.

Corollary 2.3 (Poincaré inequality at finite scale) Let $0<R_{0}<\infty$. Then for all $B=B(x, r)$ with $0<r<R_{0}$

$$
\int_{B}\left|f-f_{B}\right|^{2} d \mu \leq C_{n, 2} r^{2} \exp \left(C_{n} \sqrt{K} R_{0}\right) \int_{2 B}|\nabla f|^{2} d \mu .
$$

Remark 2.4 If the Ricci curvature of $M$ is non-negative, i.e., $K=0$, then the estimates in Theorems 2.1, 2.2 and Corollary 2.3 hold with constants independent of $0<r<\infty$. 
The Poincaré inequality yields the Sobolev inequality. We see that if $B=B(x, r)$ with $0<r<R_{0}$, then

$$
\left(\frac{1}{\mu(B)} \int_{B}|f|^{2} d \mu\right)^{1 / 2} \leq C_{n, 2} r\left(\frac{1}{\mu(B)} \int_{B}|\nabla f|^{2} d \mu\right)^{1 / 2} \text { for all } f \in C_{0}^{\infty}(B)
$$

with different $C_{n, 2}$. See [18, Thm. 5.3.3] for a more general Sobolev inequality. Hence the characterization of the bottom of the spectrum in terms of Rayleigh quotients (1.3) gives the following:

Corollary 2.5 Let $0<R_{0}<\infty$. Then there exists a constant $C>0$ depending only on $\sqrt{K} R_{0}$ and $n$ such that

$$
\lambda_{\min }(B(x, r)) \geq C r^{-2} \text { for } 0<r<R_{0} .
$$

The celebrated theorem by Grigor'yan and Saloff-Coste gives the relationship between the Poincaré inequality, the volume doubling property of the Riemannian measure, the Li-Yau Gaussian estimate for the heat kernel, and the parabolic Harnack inequality. Let $V(x, r)=\mu(B(x, r))$.

Theorem B ([18, Thm. 5.5.1, Thm. 5.5.3]) Let $0<R_{0} \leq \infty$. Consider the following conditions:

(i) (PI) There exists a constant $P_{0}>0$ such that for all $B=B(x, r)$ with $0<r<R_{0}$ and all $f \in C^{\infty}(B)$,

$$
\int_{B}\left|f-f_{B}\right|^{2} d \mu \leq P_{0} r^{2} \int_{2 B}|\nabla f|^{2} d \mu .
$$

(ii) (VD) There exists a constant $D_{0}>0$ such that for all $B=B(x, r)$ with $0<r<$ $R_{0}$

$$
\mu(2 B) \leq D_{0} \mu(B)
$$

(iii) (PHI) There exists a constant $A>0$ such that for all $B=B(x, r)$ with $0<r<R_{0}$ and all $u>0$ with $\left(\partial_{t}-\Delta\right) u=0$ in $\left(s-r^{2}, s\right) \times B$

$$
\sup _{Q_{-}} u \leq A \inf _{Q_{+}} u
$$

where $Q_{-}=\left(s-3 r^{2} / 4, s-r^{2} / 2\right) \times B(x, r / 2)$ and $Q_{+}=\left(s-r^{2} / 4, s\right) \times$ $B(x, r / 2)$.

(iv) (GE) There exists a finite constant $C>1$ such that for $0<t<R_{0}^{2}$ and $x, y \in M$,

$$
\frac{1}{C V(x, \sqrt{t})} \exp \left(-\frac{C d(x, y)^{2}}{t}\right) \leq p_{M}(t, x, y) \leq \frac{C}{V(x, \sqrt{t})} \exp \left(-\frac{d(x, y)^{2}}{C t}\right)
$$


Then

$$
(i)+(i i) \Longleftrightarrow(i i i) \Longleftrightarrow(i v)
$$

Theorem 2.1 and Corollary 2.3 assert that (i) and (ii) of Theorem B hold true for $0<R_{0}<\infty$ with constants depending only on $K, R_{0}$ and $n$. Hence, the LiYau Gaussian estimate of the heat kernel for the whole manifold $M$ and the parabolic Harnack inequality up to scale $R_{0}$ are available in our setting. Observe that the volume doubling inequality $\mu(B(x, 2 r)) \leq D_{0} \mu(B(x, r))$ implies

$$
\mu(B(x, r)) \geq C\left(\frac{r}{R}\right)^{\alpha} \mu(B(x, R)) \text { for } 0<r<R<R_{0}
$$

with $\alpha=\log D_{0} / \log 2$. We also have the following elliptic Harnack inequality since positive harmonic functions are time-independent positive solutions to the heat equation.

Corollary 2.6 (Elliptic Harnack inequality) Let $0<r_{1}<r_{2}<R_{0}<\infty$. If $h$ is $a$ positive harmonic function in $B\left(x, r_{2}\right)$, then

$$
C^{-1} \leq \frac{h(y)}{h(x)} \leq C \quad \text { for } y \in B\left(x, r_{1}\right)
$$

where $C>1$ depends only on $\sqrt{K} R_{0}, r_{1} / r_{2}$ and $n$.

\section{Torsion Function and the Bottom of Spectrum}

In this section we obtain estimates between the bottom of the spectrum and the torsion function $v_{D}$. We shall prove the second and the third inequalities of (1.5).

Since the Green function $G_{D}(x, y)$ is the integral of the heat kernel $p_{D}(t, x, y)$ with respect to $t \in(0, \infty)$, we have

$$
v_{D}(x)=\int_{0}^{\infty} P_{D}(t, x) d t
$$

where

$$
P_{D}(t, x)=\int_{D} p_{D}(t, x, y) d \mu(y) .
$$

We note that $P_{D}(t, x)=\mathbb{P}_{x}\left[\tau_{D}>t\right]$, i.e., the survival probability that the Brownian motion $\left(B_{t}\right)_{t \geq 0}$ started at $x$ stays in $D$ up to time $t$, where $\tau_{D}$ is the first exit time from 
$D$. We also observe that $P_{D}(t, x)$ is considered to be the (weak) solution to

$$
\begin{aligned}
\left(\frac{\partial}{\partial t}-\Delta\right) u(t, x)=0 & \text { in }(0, \infty) \times D, \\
u(t, x)=0 & \text { on }(0, \infty) \times \partial D, \\
u(0, x)=1 & \text { on }\{0\} \times D .
\end{aligned}
$$

Let $\pi_{D}(t)=\sup _{x \in D} P_{D}(t, x)$. Let us begin with the proof of the second inequality of (1.5).

Lemma 3.1 If $\lambda_{\min }(D)>0$, then $\lambda_{\min }(D)\left\|v_{D}\right\|_{\infty} \geq 1$.

Proof We follow [1, Lem. 3.2, Lem. 3.3]. Without loss of generality we may assume that $\left\|v_{D}\right\|_{\infty}<\infty$. It suffices to show the following two estimates:

$$
\begin{aligned}
& \exp \left(-\lambda_{\min }(D) t\right) \leq \pi_{D}(t) \text { for all } t>0 \\
& \text { If } C>1 \text {, then } \pi_{D}(t) \leq \frac{C}{C-1} \exp \left(-\frac{t}{C\left\|v_{D}\right\|_{\infty}}\right) \text { for all } t>0 .
\end{aligned}
$$

In fact, we obtain from (3.1) and (3.2) that

$$
\exp \left(-\lambda_{\min }(D) t+\frac{t}{C\left\|v_{D}\right\|_{\infty}}\right) \leq \frac{C}{C-1}
$$

which holds for all $t>0$ only if

$$
\lambda_{\min }(D) \geq \frac{1}{C\left\|v_{D}\right\|_{\infty}} .
$$

Since $C>1$ is arbitrary, we have $\lambda_{\min }(D)\left\|v_{D}\right\|_{\infty} \geq 1$.

Let us prove (3.1). Take $\alpha>\lambda_{\min }(D)$. Then we find $\varphi \in C_{0}^{\infty}(D)$ such that $\|\nabla \varphi\|_{2}^{2} /\|\varphi\|_{2}^{2} \leq \alpha$. Take a bounded domain $\Omega$ such that $\operatorname{supp} \varphi \subset \Omega \subset D$. Then $\Omega$ has no essential spectrum. Let $\lambda_{\Omega}$ and $\varphi_{\Omega}$ be the first eigenvalue and its positive eigenfunction with $\left\|\varphi_{\Omega}\right\|_{2}=1$ for $\Omega$, respectively. By definition

$$
\lambda_{\Omega}=\inf \left\{\frac{\|\nabla \psi\|_{2}^{2}}{\|\psi\|_{2}^{2}}: \psi \in C_{0}^{\infty}(\Omega)\right\} \leq \frac{\|\nabla \varphi\|_{2}^{2}}{\|\varphi\|_{2}^{2}} \leq \alpha
$$

Since $u(t, x)=\exp \left(-\lambda_{\Omega} t\right) \varphi_{\Omega}(x)$ is the solution to the heat equation in $(0, \infty) \times \Omega$ such that $u(0, x)=\varphi_{\Omega}(x)$ and $u(t, x)=0$ on $(0, \infty) \times \partial \Omega$, it follows from the comparison principle that

$\exp \left(-\lambda_{\Omega} t\right) \varphi_{\Omega}(x) \leq \int_{\Omega} p_{D}(t, x, y) \varphi_{\Omega}(y) d \mu(y) \leq\left\|\varphi_{\Omega}\right\|_{\infty} P_{D}(t, x) \leq\left\|\varphi_{\Omega}\right\|_{\infty} \pi_{D}(t)$ 
in $(0, \infty) \times \Omega$. Taking the supremum for $x \in \Omega$, and then dividing by $0<\left\|\varphi_{\Omega}\right\|_{\infty}<$ $\infty$, we obtain

$$
\exp (-\alpha t) \leq \exp \left(-\lambda_{\Omega} t\right) \leq \pi_{D}(t)
$$

Since $\alpha>\lambda_{\min }(D)$ is arbitrary, we have (3.1).

Let us prove (3.2) to complete the proof of the lemma. Let $C>1$ and $\beta=$ $1 /\left(C\left\|v_{D}\right\|_{\infty}\right)$. Put

$$
w(t, x)=e^{-\beta t}\left(v_{D}(x)+(C-1)\left\|v_{D}\right\|_{\infty}\right) .
$$

Since $-\Delta v_{D}=1$ in $D$, it follows that

$$
\begin{aligned}
\left(\frac{\partial}{\partial t}-\Delta\right) w & =-\beta e^{-\beta t}\left(v_{D}+(C-1)\left\|v_{D}\right\|_{\infty}\right)-e^{-\beta t} \Delta v_{D} \\
& =e^{-\beta t}\left(-\frac{v_{D}+(C-1)\left\|v_{D}\right\|_{\infty}}{C\left\|v_{D}\right\|_{\infty}}+1\right) \\
& \geq e^{-\beta t}\left(-\frac{\left\|v_{D}\right\|_{\infty}+(C-1)\left\|v_{D}\right\|_{\infty}}{C\left\|v_{D}\right\|_{\infty}}+1\right)=0 .
\end{aligned}
$$

Hence $w$ is a super solution to the heat equation. By the comparison principle

$$
\begin{aligned}
(C-1)\left\|v_{D}\right\|_{\infty} P_{D}(t, x) & \leq w(t, x) \\
& =e^{-\beta t}\left(v_{D}(x)+(C-1)\left\|v_{D}\right\|_{\infty}\right) \leq C e^{-\beta t}\left\|v_{D}\right\|_{\infty} .
\end{aligned}
$$

Dividing the inequality by $0<\left\|v_{D}\right\|_{\infty}<\infty$, and taking the supremum for $x \in D$, we obtain (3.2).

Next we prove the third inequality of (1.5) under an additional assumption on $\lambda_{\min }(D)$.

Lemma 3.2 There exist $\Lambda_{0}>0$ and $C_{0}>0$ depending only on $K$ and $n$ such that if either $\lambda_{\min }(D)>\Lambda_{0}$ or $\left\|v_{D}\right\|_{\infty}<1 / \Lambda_{0}$, then

$$
\lambda_{\min }(D)\left\|v_{D}\right\|_{\infty} \leq C_{0} .
$$

Proof In view of Lemma 3.1, we see that $\left\|v_{D}\right\|_{\infty}<1 / \Lambda_{0}$ implies $\lambda_{\min }(D)>\Lambda_{0}$. So, it suffices to show (3.3) under the assumption $\lambda_{\min }(D)>\Lambda_{0}$ with $\Lambda_{0}$ to be determined later.

For simplicity we write $\lambda_{D}$ for $\lambda_{\min }(D)$, albeit $\lambda_{\min }(D)$ need not be an eigenvalue. Let $0<R_{0}<\infty$. By symmetry, the Gaussian estimate (2.1) implies

$$
\begin{gathered}
\frac{1}{C V(x, \sqrt{t})^{1 / 2} V(y, \sqrt{t})^{1 / 2}} \exp \left(-\frac{C d(x, y)^{2}}{t}\right) \leq p_{M}(t, x, y) \\
\leq \frac{C}{V(x, \sqrt{t})^{1 / 2} V(y, \sqrt{t})^{1 / 2}} \exp \left(-\frac{d(x, y)^{2}}{C t}\right)
\end{gathered}
$$


with the same $C$; and conversely, (3.4) implies (2.1) with different $C$ depending only on $\sqrt{K} R_{0}$ and $n$ by volume doubling. Let $0<t<R_{0}^{2}$. By [14, Ex. 10.29] we have

$$
\begin{aligned}
p_{D}(t, x, y) & \leq p_{D}(t, x, y)^{1 / 2} p_{M}(t, x, y)^{1 / 2} \\
& \leq\left(e^{-\lambda D} \sqrt{p_{D}(t / 2, x, x) p_{D}(t / 2, y, y)}\right)^{1 / 2} p_{M}(t, x, y)^{1 / 2} \\
& \leq e^{-\lambda_{D} t / 4} p_{M}(t / 2, x, x)^{1 / 4} p_{M}(t / 2, y, y)^{1 / 4} p_{M}(t, x, y)^{1 / 2},
\end{aligned}
$$

so that the upper estimates of (2.1) and (3.4), together with volume doubling, show that $p_{D}(t, x, y)$ is bounded by

$$
\begin{aligned}
& e^{-\lambda_{D} t / 4}\left\{\frac{C}{V(x, \sqrt{t / 2})}\right\}^{1 / 4} \cdot\left\{\frac{C}{V(y, \sqrt{t / 2})}\right\}^{1 / 4} \cdot \\
& \left\{\frac{C}{V(x, \sqrt{t})^{1 / 2} V(y, \sqrt{t})^{1 / 2}} \exp \left(-\frac{d(x, y)^{2}}{C t}\right)\right\}^{1 / 2} \\
& \leq e^{-\lambda_{D} t / 4} \frac{C C^{\prime}}{V(x, \sqrt{t})^{1 / 2} V(y, \sqrt{t})^{1 / 2}} \exp \left(-\frac{d(x, y)^{2}}{2 C t}\right),
\end{aligned}
$$

where $C^{\prime}$ takes care of the various volume doubling factors. By the lower estimate of (3.4) with $2 C^{2} t$ in place of $t$ and volume doubling, we find $C_{1} \geq 1$ depending only on $\sqrt{K} R_{0}$ and $n$ such that

$$
p_{D}(t, x, y) \leq C_{1} e^{-\lambda_{D} t / 4} p_{M}\left(2 C^{2} t, x, y\right)
$$

Integrating the inequality with respect to $y \in D$, we obtain

$$
\begin{aligned}
P_{D}(t, x) & =\int_{D} p_{D}(t, x, y) d \mu(y) \\
& \leq C_{1} e^{-\lambda_{D} t / 4} \int_{D} p_{M}\left(2 C^{2} t, x, y\right) d \mu(y) \leq C_{1} e^{-\lambda_{D} t / 4} .
\end{aligned}
$$

Taking the supremum over $x \in D$, we obtain

$$
\pi_{D}(t) \leq C_{1} \exp \left(-\frac{\lambda_{D} t}{4}\right) \text { for } 0<t<R_{0}^{2} .
$$

Let $T=R_{0}^{2} / 2$. We claim that (3.3) holds with $C_{0}=8 \log \left(2 C_{1}\right)$, and with $\Lambda_{0}=$ $4 T^{-1} \log \left(2 C_{1}\right)$ or

$$
C_{1} \exp \left(-\frac{\Lambda_{0} T}{4}\right)=\frac{1}{2} .
$$

Suppose $\lambda_{D}>\Lambda_{0}$. Then (3.5) with $t=T$ yields $\pi_{D}(T) \leq 1 / 2$. Solving the initial value problem from time $T$, we see that

$$
P_{D}(t, x) \leq \pi_{D}(T) \cdot P_{D}(t-T, x) \leq \frac{1}{2} \quad \text { for } t \geq T .
$$


Take the supremum for $x \in D$. We find

$$
\pi_{D}(t) \leq \frac{1}{2} \text { for } t \geq T
$$

Repeating the same argument, we obtain

$$
\pi_{D}(t) \leq \frac{1}{2^{k}} \text { for } k T \leq t<(k+1) T \text { with } k=0,1,2, \ldots
$$

Hence

$$
\begin{aligned}
v_{D}(x) & =\int_{0}^{\infty} P_{D}(t, x) \mathrm{d} t=\sum_{k=0}^{\infty} \int_{k T}^{(k+1) T} P_{D}(t, x) \mathrm{d} t \\
& \leq \sum_{k=0}^{\infty} \int_{k T}^{(k+1) T} \pi_{D}(t) \mathrm{d} t \leq T \sum_{k=0}^{\infty} \frac{1}{2^{k}}=2 T \leq \frac{2 \Lambda_{0} T}{\lambda_{D}}=\frac{8 \log \left(2 C_{1}\right)}{\lambda_{D}}
\end{aligned}
$$

by (3.6). Taking the supremum for $x \in D$, we obtain $\lambda_{D}\left\|v_{D}\right\|_{\infty} \leq 8 \log \left(2 C_{1}\right)$, as required.

Remark 3.3 If the Gaussian estimate (2.1) holds uniformly for all $0<t<\infty$, then there exists $C>0$ such that $\lambda_{\min }(D)\left\|v_{D}\right\|_{\infty} \leq C$ for all $D \subset M$. This is the case when $K=0$. See [19].

\section{Capacitary Width and Harmonic Measure}

By $\omega^{x}(E, D)$ we denote the harmonic measure of $E$ in $D$ evaluated at $x$. In this section we give an estimate for harmonic measure in terms of capacitary width. This will be crucial for the proof of Theorem 1.3.

Theorem 4.1 (cf. [1, Thm. 12.7]) Let $0<R_{0}<\infty$. Let $D \subset M$ be an open set with $w_{\eta}(D)<R_{0}$. If $x \in D$ and $R>0$, then

$$
\omega^{x}(D \cap \partial B(x, R), D \cap B(x, R)) \leq \exp \left(2 C_{2}-\frac{C_{2} R}{w_{\eta}(D)}\right),
$$

where $C_{2}$ depends only on $\sqrt{K} R_{0}, \eta$ and $n$.

Let us begin by estimating the torsion function of a ball.

Lemma 4.2 Let $0<R_{0}<\infty$. Then there exists a constant $C>1$ depending only on $\sqrt{K} R_{0}$ and $n$ such that

$$
C^{-1} r^{2} \leq\left\|v_{B(x, r)}\right\|_{\infty} \leq C r^{2} \text { for } 0<r<R_{0}
$$


Proof Let $0<r<R_{0}$. Write $B=B(x, r)$ for simplicity. We have $\lambda_{\min }(B) \geq C r^{-2}$ by Corollary 2.5. Since $B$ is bounded, the bottom of the spectrum is an eigenvalue. So let us write $\lambda_{B}$ for $\lambda_{\min }(B)$. Let $z \in B$. In view of [14, Ex. 10.29], the Gaussian estimate (2.1) and the volume doubling property, we have

$$
\begin{aligned}
v_{B}(z) & =\int_{B} G_{B}(z, y) d \mu(y)=\int_{0}^{\infty} d t \int_{B} p_{B}(t, z, y) d \mu(y) \\
& =\int_{0}^{r^{2}} d t \int_{B} p_{B}(t, z, y) d \mu(y)+\int_{r^{2}}^{\infty} d t \int_{B} p_{B}(t, z, y) d \mu(y) \\
& \leq r^{2}+\int_{r^{2}}^{\infty} e^{-\lambda_{B}\left(t-r^{2}\right)} d t \int_{B} \sqrt{p_{B}\left(r^{2}, z, z\right) p_{B}\left(r^{2}, y, y\right)} d \mu(y) \\
& \leq r^{2}+\frac{1}{\lambda_{B}} \int_{B} \frac{C d \mu(y)}{\sqrt{V(z, r) V(y, r)}} \leq r^{2}+C r^{2},
\end{aligned}
$$

where $C$ depends only on $\sqrt{K} R_{0}$ and $n$. Hence $\left\|v_{B}\right\|_{\infty} \leq C r^{2}$.

The opposite inequality is an immediate consequence of the combination of Corollary 2.5 and Lemma 3.1. But for later purpose we give a direct proof based on a lower estimate of the Dirichlet heat kernel of a ball: if $x \in M$, then

$$
p_{B}(t, y, z) \geq \frac{C}{V(x, \sqrt{t})} \text { for } y, z \in \varepsilon B \text { and } 0<t<\varepsilon r^{2}
$$

valid for some $0<\varepsilon<1$ and $C>0$. In fact, this lower estimate is equivalent to the Gaussian estimate (2.1). See e.g. [6, (1.5)]. If $y \in \varepsilon B$, then

$v_{B}(y)=\int_{B} G_{B}(y, z) d \mu(z) \geq \int_{0}^{\varepsilon r^{2}} d t \int_{\varepsilon B} p_{B}(t, y, z) d \mu(z) \geq \frac{\varepsilon r^{2} C \mu(\varepsilon B)}{V(x, \sqrt{\varepsilon} r)} \geq C r^{2}$

by volume doubling. Thus $\left\|v_{B}\right\|_{\infty} \geq C r^{2}$.

For later use we record the above estimate: if $0<r<R_{0}$, then

$$
v_{B(x, r)} \geq C_{3} r^{2} \text { on } B(x, \varepsilon r)
$$

where $\varepsilon$ and $C_{3}$ depends only on $\sqrt{K} R_{0}$ and $n$.

Remark 4.3 In case $K>0$, the inequality (4.1) does not necessarily hold for all $0<$ $r<\infty$ uniformly. Let $\mathbb{H}^{n}$ be the $n$-dimensional hyperbolic space of constant curvature -1 . Then the torsion function for $B(a, r)$ is a radial function $f(\rho)$ of $\rho=d(x, a)$ satisfying

$$
-1=\Delta f(\rho)=\frac{1}{(\sinh \rho)^{n-1}} \frac{d}{d \rho}\left\{(\sinh \rho)^{n-1} \frac{d f}{d \rho}\right\} \text { for } 0<\rho<r
$$


$f(r)=0, f^{\prime}(0)=0$ and $f(0)=\left\|v_{B(a, r)}\right\|_{\infty}$. See [11, pp. 176-177] or [14, (3.85)]. Hence

$$
\left\|v_{B(a, r)}\right\|_{\infty}=\int_{0}^{r} \int_{0}^{\rho}\left(\frac{\sinh t}{\sinh \rho}\right)^{n-1} d t d \rho .
$$

Since the integrand is less than 1 , we have $\left\|v_{B(a, r)}\right\|_{\infty} \leq \frac{1}{2} r^{2}$ for all $r>0$. Observe that $t \leq \sinh t$ for $t>0$ and $\sinh \rho \leq \rho \cosh R_{0}$ for $0<\rho<R_{0}$. Hence, if $0<r<R_{0}$, then

$$
\left\|v_{B(a, r)}\right\|_{\infty} \geq \int_{0}^{r} \int_{0}^{\rho}\left(\frac{t}{\rho \cosh R_{0}}\right)^{n-1} d t d \rho=\frac{r^{2}}{2 n\left(\cosh R_{0}\right)^{n-1}},
$$

so that $\left\|v_{B(a, r)}\right\|_{\infty} \approx r^{2}$. This gives the estimate in Lemma 4.2 with explicit bounds.

On the other hand, if $r>1$, then $\sinh \rho \geq \frac{1}{2}\left(1-e^{-2}\right) e^{\rho}$ for $1<\rho<r$, so that

$$
\begin{aligned}
\| v_{B(a, r) \|_{\infty}} & \leq \int_{0}^{1} \int_{0}^{\rho} d t d \rho+\int_{1}^{r} \int_{0}^{\rho}\left(\frac{\sinh t}{\sinh \rho}\right)^{n-1} d t d \rho \\
& \leq \frac{1}{2}+\int_{1}^{r} \int_{0}^{\rho}\left(\frac{e^{t}}{\left(1-e^{-2}\right) e^{\rho}}\right)^{n-1} d t d \rho \\
& =\frac{1}{2}+\frac{1}{n-1} \int_{1}^{r} \frac{e^{(n-1) \rho}-1}{\left(\left(1-e^{-2}\right) e^{\rho}\right)^{n-1}} d \rho \leq \frac{1}{2}+\frac{r-1}{(n-1)\left(1-e^{-2}\right)^{n-1}} .
\end{aligned}
$$

Thus $\left\|v_{B(a, r)}\right\|_{\infty}=O(r)$ as $r \rightarrow \infty$, so (4.1) fails to hold uniformly for $0<r<\infty$. This example illustrates that the assumption $0<r<R_{0}$ cannot be dropped in Lemma 4.2.

Next we compare capacity and volume. Observe that $\operatorname{Cap}_{D}(E)$ coincides with the Green capacity of $E$ with respect to $D$, i.e.,

$$
\operatorname{Cap}_{D}(E)=\sup \left\{\|v\|: \operatorname{supp} v \subset E \text { and } \int_{D} G_{D}(x, y) d v(y) \leq 1 \text { on } D\right\}
$$

where $\|v\|$ stands for the total mass of the measure $v$.

Lemma 4.4 Let $0<R_{0}<\infty$. There exists a constant $C_{4}>0$ depending only on $\sqrt{K} R_{0}$ and $n$ such that if $0<r<R_{0}$, then

$$
\frac{\mu(E)}{\mu(\bar{B}(x, r))} \leq C_{4} \frac{\operatorname{Cap}_{B(x, 2 r)}(E)}{\operatorname{Cap}_{B(x, 2 r)}(\bar{B}(x, r))}
$$

for every Borel set $E \subset \bar{B}(x, r)$. 
Proof Let $0<r<R_{0}$. Lemma 4.2 yields

$$
\begin{aligned}
\int_{E} G_{B(x, 2 r)}(y, z) d \mu(z) & \leq \int_{B(x, 2 r)} G_{B(x, 2 r)}(y, z) d \mu(z) \leq\left\|v_{B(x, 2 r)}\right\|_{\infty} \\
& \leq C r^{2} \text { for all } y \in M,
\end{aligned}
$$

where $C$ depends only on $\sqrt{K} R_{0}$ and $n$. Hence the characterization (4.2) of capacity gives

$$
\operatorname{Cap}_{B(x, 2 r)}(E) \geq \frac{\mu(E)}{C r^{2}} .
$$

Let $\varphi(y)=\min \{2-d(y, x) / r, 1\}$. Observe that $\varphi \in W_{0}^{1}(B(x, 2 r)),|\nabla \varphi| \leq 1 / r$ and $\varphi=1$ on $\bar{B}(x, r)$. The definition of capacity and the volume doubling property yield

$$
\operatorname{Cap}_{B(x, 2 r)}(\bar{B}(x, r)) \leq \int_{B(x, 2 r)}|\nabla \varphi|^{2} d \mu \leq \frac{\mu(\bar{B}(x, 2 r))}{r^{2}} \leq \frac{C \mu(\bar{B}(x, r))}{r^{2}} .
$$

This, together with (4.3) for $E=\bar{B}(x, r)$, shows that $\operatorname{Cap}_{B(x, 2 r)}(\bar{B}(x, r)) \approx$ $r^{-2} \mu(\bar{B}(x, r))$ with the constant of comparison depending only on $\sqrt{K} R_{0}$ and $n$. Dividing (4.3) by $\operatorname{Cap}_{B(x, 2 r)}(\bar{B}(x, r))$, we obtain the lemma.

Let us introduce regularized reduced functions, which are closely related to capacity and harmonic measure. See [3, Sect. 5.3-7] for the Euclidean case. Let $D$ be an open set. For $E \subset D$ and a non-negative function $u$ in $E$, we define the reduced function ${ }^{D} \mathbf{R}_{u}^{E}$ by

${ }^{D} \mathbf{R}_{u}^{E}(x)=\inf \{v(x): v \geq 0$ is superharmonic in $D$ and $v \geq u$ on $E\}$ for $x \in D$.

The lower semicontinuous regularization of ${ }^{D} \mathbf{R}_{u}^{E}$ is called the regularized reduced function or balayage and is denoted by ${ }^{D} \widehat{\mathbf{R}}_{u}^{E}$. It is known that ${ }^{D} \widehat{\mathbf{R}}_{u}^{E}$ is a non-negative superharmonic function, ${ }^{D} \widehat{\mathbf{R}}_{u}^{E} \leq{ }^{D} \mathbf{R}_{u}^{E}$ in $D$ with equality outside a polar set. If $u$ is a non-negative superharmonic function in $D$, then ${ }^{D} \widehat{\mathbf{R}}_{u}^{E} \leq u$ in $D$. By the maximum principle ${ }^{D} \widehat{\mathbf{R}}_{u}^{E}$ is non-decreasing with respect to $D$ and $E$. If $u$ is the constant function 1 , then ${ }^{D} \widehat{\mathbf{R}}_{1}^{E}(x)$ is the probability of Brownian motion hitting $E$ before leaving $D$ when it starts at $x$. In an almost verbatim way we can extend $[1, \mathrm{Lem}$. F] to the present setting. But, for completeness, we shall provide a proof.

Lemma 4.5 Let $0<r<R<R_{0}<\infty$.

(i) $\inf _{\bar{B}(x, r)}^{B(x, R)} \widehat{\mathbf{R}}_{1}^{E} \leq \frac{\operatorname{Cap}_{B(x, R)}(E)}{\operatorname{Cap}_{B(x, R)}(\bar{B}(x, r))}$ for $E \subset B(x, R)$.

(ii) $\frac{\operatorname{Cap}_{B(x, R)}(E)}{\operatorname{Cap}_{B(x, R)}(\bar{B}(x, r))} \leq C \inf _{\bar{B}(x, r)}^{B(x, R)} \widehat{\mathbf{R}}_{1}^{E} \quad$ for $E \subset \bar{B}(x, r)$ with $C>1$ depending only on $\sqrt{K} R_{0}, r / R$ and $n$. 
Proof Let $v_{E}$ and $v_{B}$ be the capacitary measures of $E$ and $\bar{B}(x, r)$, respectively. Then $v_{E}$ is supported on $\bar{E}, G_{B(x, R)} v_{E}=B(x, R) \widehat{\mathbf{R}}_{1}^{E}$ and $\left\|v_{E}\right\|=\operatorname{Cap}_{B(x, R)}(E) ; v_{B}$ is supported on $\bar{B}(x, r), G_{B(x, R)} v_{B}={ }^{B(x, R)} \widehat{\mathbf{R}}_{1}^{\bar{B}(x, r)}$ and $\left\|v_{B}\right\|=\operatorname{Cap}_{B(x, R)}(\bar{B}(x, r))$. In particular, $G_{B(x, R)} v_{B} \leq 1$ in $B(x, R)$ and hence

$$
\begin{aligned}
\operatorname{Cap}_{B(x, R)}(E) & \geq \int G_{B(x, R)} v_{B} d v_{E}=\int G_{B(x, R)} v_{E} d v_{B} \\
& =\int B(x, R) \widehat{\mathbf{R}}_{1}^{E} d v_{B} \geq \int\left(\inf _{\bar{B}(x, r)}^{B(x, R)} \widehat{\mathbf{R}}_{1}^{E}\right) d v_{B} \\
& =\left(\inf _{B(x, r)}^{B(x, R)} \widehat{\mathbf{R}}_{1}^{E}\right) \operatorname{Cap}_{B(x, R)}(\bar{B}(x, r)) .
\end{aligned}
$$

Thus (i) follows.

Let $\rho=(r+R) / 2$. The elliptic Harnack inequality (Corollary 2.6) implies

$$
\begin{aligned}
& G_{B(x, R)}(z, y) \approx G_{B(x, R)}(z, x) \text { for } z \in \partial B(x, \rho) \text { and } y \in \bar{B}(x, r), \\
& B(x, R) \widehat{\mathbf{R}}_{1}^{\bar{B}(x, r)} \approx 1 \text { on } \partial B(x, \rho),
\end{aligned}
$$

where, and hereafter, the constants of comparison depend only on $\sqrt{K} R_{0}, r / R$ and $n$. Let $E \subset \bar{B}(x, r)$. Since supp $v_{E} \subset \bar{B}(x, r)$, we have for $z \in \partial B(x, \rho)$,

$$
\begin{aligned}
& B(x, R) \widehat{\mathbf{R}}_{1}^{E}(z)=\int G_{B(x, R)}(z, y) d v_{E}(y) \approx G_{B(x, R)}(z, x) \operatorname{Cap}_{B(x, R)}(E), \\
& { }^{B(x, R)} \widehat{\mathbf{R}}_{1}^{\bar{B}(x, r)}(z)=\int G_{B(x, R)}(z, y) d v_{B}(y) \approx G_{B(x, R)}(z, x) \operatorname{Cap}_{B(x, R)}(\bar{B}(x, r)),
\end{aligned}
$$

so that

$$
\frac{\operatorname{Cap}_{B(x, R)}(E)}{\operatorname{Cap}_{B(x, R)}(\bar{B}(x, r))} \approx B(x, R) \widehat{\mathbf{R}}_{1}^{E}(z) .
$$

Since $z \in \partial B(x, \rho)$ is arbitrary, the superharmonicity of ${ }^{B(x, R)} \widehat{\mathbf{R}}_{1}^{E}$ and the maximum principle yield (ii).

We restate the above lemma in terms of harmonic measure. We recall $\omega^{x}(E, D)$ stands for the harmonic measure of $E$ in $D$ evaluated at $x$. We see that if $E$ is a compact subset of $B(x, R)$, then

$$
\omega(\partial B(x, R), B(x, R) \backslash E)=1-{ }^{B(x, R)} \widehat{\mathbf{R}}_{1}^{E} \quad \text { on } B(x, R) .
$$

Strictly speaking, the harmonic measure is extended by the right-hand side. Lemma 4.5 reads as follows.

Lemma 4.6 Let $0<r<R<R_{0}<\infty$. 
(i) $1-\frac{\operatorname{Cap}_{B(x, R)}(E)}{\operatorname{Cap}_{B(x, R)}(\bar{B}(x, r))} \leq \sup _{\bar{B}(x, r)} \omega(\partial B(x, R), B(x, R) \backslash E) \quad$ for $E \subset B(x, R)$.

(ii) $\sup _{\bar{B}(x, r)} \omega(\partial B(x, R), B(x, R) \backslash E) \leq 1-C^{-1} \frac{\operatorname{Cap}_{B(x, R)}(E)}{\operatorname{Cap}_{B(x, R)}(\bar{B}(x, r))}$ for $E \subset \bar{B}(x, r)$ with $C>1$ depending only on $\sqrt{K} R_{0}, r / R$ and $n$. In particular, if $0<r<R_{0} / 2$, then

$$
\sup _{\bar{B}(x, r)} \omega(\partial B(x, 2 r), B(x, 2 r) \backslash E) \leq 1-C_{5}^{-1} \frac{\operatorname{Cap}_{B(x, 2 r)}(E)}{\operatorname{Cap}_{B(x, 2 r)}(\bar{B}(x, r))},
$$

where $C_{5}>1$ depends only on $\sqrt{K} R_{0}$ and $n$.

Applying Lemma 4.6 repeatedly, we obtain the following estimate of harmonic measure, which is a preliminary version of Theorem 4.1.

Lemma 4.7 Let $0<R_{0}<\infty$. Let $D \subset M$ be an open set with $w_{\eta}(D)<R_{0}$. Suppose $x \in D$ and $R>0$. If $k$ is a non-negative integer such that $R-2 k w_{\eta}(D)>0$, then

$$
\sup _{D \cap \bar{B}\left(x, R-2 k w_{\eta}(D)\right)} \omega(D \cap \partial B(x, R), D \cap B(x, R)) \leq\left(1-C_{5}^{-1} \eta\right)^{k} .
$$

Proof For simplicity let $\omega_{0}=\omega(D \cap \partial B(x, R), D \cap B(x, R))$. By definition we find $r>w_{\eta}(D)$ arbitrarily close to $w_{\eta}(D)$ such that

$$
\frac{\operatorname{Cap}_{B(y, 2 r)}(\bar{B}(y, r) \backslash D)}{\operatorname{Cap}_{B(y, 2 r)}(\bar{B}(y, r))} \geq \eta \text { for all } y \in D
$$

Hence it suffices to show that $\omega_{0} \leq\left(1-C_{5}^{-1} \eta\right)^{k}$ in $D \cap \bar{B}(x, R-2 k r)$. Let us prove this inequality by induction on $k$. The case $k=0$ holds trivially. Let $k \geq 1$ and suppose $\omega_{0} \leq\left(1-C_{5}^{-1} \eta\right)^{k-1}$ in $D \cap \bar{B}(x, R-2(k-1) r)$. Take $y \in D \cap \partial B(x, R-2 k r)$ and let $E=\bar{B}(y, r) \backslash D$. Since $D \cap B(y, 2 r) \subset D \cap \bar{B}(x, R-2(k-1) r)$, we have

$$
\begin{aligned}
\omega_{0} & \leq\left(1-C_{5}^{-1} \eta\right)^{k-1} \omega(D \cap \partial B(y, 2 r), D \cap B(y, 2 r)) \\
& \leq\left(1-C_{5}^{-1} \eta\right)^{k-1} \omega(\partial B(y, 2 r), D \backslash E) \leq\left(1-C_{5}^{-1} \eta\right)^{k}
\end{aligned}
$$

in $D \cap B(y, 2 r)$. Since $y \in D \cap \partial B(x, R-2 k r)$ is arbitrary, we have $\omega_{0} \leq\left(1-C_{5}^{-1} \eta\right)^{k}$ on $D \cap \partial B(x, R-2 k r)$, and hence in $D \cap \bar{B}(x, R-2 k r)$ by the maximum principle, as required.

This lemma and the definition of capacitary width yield 
Proof of Theorem 4.1 Let $k$ be the integer such that $2 k w_{\eta}(D)<R \leq 2(k+1) w_{\eta}(D)$. Lemma 4.7 gives

$$
\begin{aligned}
\omega^{x}(D \cap \partial B(x, R), D \cap B(x, R)) & \leq\left(1-C_{5}^{-1} \eta\right)^{k}=\exp \left(-k \log \frac{1}{1-C_{5}^{-1} \eta}\right) \\
& \leq \exp \left(-\left(\frac{R}{2 w_{\eta}(D)}-1\right) \log \frac{1}{1-C_{5}^{-1} \eta}\right),
\end{aligned}
$$

which implies the required inequality with

$$
C_{2}=\frac{1}{2} \log \frac{1}{1-C_{5}^{-1} \eta}
$$

\section{Proofs of Theorems 1.3 and 1.6}

In this section we prove Theorem 1.3 and complete the proof of Theorem 1.6 by showing

Theorem 5.1 Let $0<R_{0}<\infty$. If $w_{\eta}(D)<R_{0}$, then

$$
C^{-1} w_{\eta}(D)^{2} \leq\left\|v_{D}\right\|_{\infty} \leq C w_{\eta}(D)^{2}
$$

where $C$ depends only on $\sqrt{K} R_{0}, \eta$ and $n$.

This theorem, together with (3.2) in Lemma 3.1, immediately yields the following estimate of the survival probability, which plays a crucial role in the proof of Theorem 1.5.

Theorem 5.2 Let $0<R_{0}<\infty$. There exist positive constants $C_{6}$ and $C_{7}$ depending only on $\sqrt{K} R_{0}, \eta$ and $n$ such that

$$
P_{D}(t, x) \leq C_{6} \exp \left(-\frac{C_{7} t}{w_{\eta}(D)^{2}}\right) \text { for all } t>0 \text { and } x \in D,
$$

whenever $w_{\eta}(D)<R_{0}$.

Let us begin with a uniform estimate of the capacity of balls.

Lemma 5.3 Let $0<R_{0}<\infty$. For $0<t \leq 1$, define

$$
\kappa(t)=\inf \left\{\frac{\operatorname{Cap}_{B(x, 2 R)}(\bar{B}(x, t R))}{\operatorname{Cap}_{B(x, 2 R)}(\bar{B}(x, R))}: x \in M, 0<R<R_{0}\right\} .
$$

Then $\lim _{t \rightarrow 1} \kappa(t)=1$. 
Proof Without loss of generality we may assume that $1 / 2<t \leq 1$. Let $\Omega=$ $B(x, 2 R) \backslash \bar{B}(x, t R)$ and let $E_{t}=\partial B(x, t R)$. We find $a>0$ such that for each $y \in E_{t}$ and $0<r<\frac{1}{4} R$ there exists a ball of radius ar lying in $B(y, r) \backslash \Omega$. This means that

$$
\frac{\mu(B(y, r) \backslash \Omega)}{\mu(B(y, r))} \geq \varepsilon
$$

with some $\varepsilon>0$ depending only on $a$ and the doubling constant. By Lemmas 4.4 and 4.6 we have

$$
\sup _{\bar{B}(y, r)} \omega(\partial B(y, 2 r), B(y, 2 r) \cap \Omega) \leq 1-\varepsilon^{\prime}
$$

with $\varepsilon^{\prime}>0$ independent of $x, R, t, y$ and $r$.

The technique in the proof of [2, Thm. 1] yields a positive superharmonic function $s$ in $\Omega$ such that

$$
s \approx \operatorname{dist}\left(\cdot, E_{t}\right)^{\alpha}
$$

where $\alpha>0$ and the constants of comparison are independent of $x, R$ and $t$. In fact, let $r_{k}=4^{k}, k \in \mathbb{Z}$. For each $k \in \mathbb{Z}$ choose a locally finite covering of $E_{t}$ by open balls $B\left(x_{k j}, r_{k} / 4\right), j \in J_{k}$; let $B_{k j}=B\left(x_{k j}, r_{k}\right)$. By (5.3) we find a positive continuous function $u_{k j}$ in $\Omega \cap \bar{B}_{k j}$, superharmonic in $\Omega \cap B_{k j}$, such that $\varepsilon^{\prime \prime} \leq u_{k j} \leq 2$ in $\Omega \cap B_{k j}$, $u_{k j} \geq 1$ in $\Omega \cap \partial B_{k j}, u_{k j} \leq 1-\varepsilon^{\prime \prime}$ in $\Omega \cap \frac{1}{2} B_{k j}$, where $\varepsilon^{\prime \prime}$ is a small positive constant depending only on $\varepsilon^{\prime}$. Let $A=1-\varepsilon^{\prime \prime} / 2$ and extend $u_{k j}$ on $\Omega \backslash \bar{B}_{k j}$ by $u_{k j}=\infty$. Then

$$
s(x)=\inf \left\{A^{-k} u_{k j}(x): k \in \mathbb{Z}, j \in J_{k}\right\}, \quad x \in \Omega
$$

is a superharmonic function in $\Omega$ satisfying (5.4) with $\alpha=|\log A| / \log 4$. Actually, we can make $s$ a strong barrier. In the present context, however, superharmonicity is enough.

From (5.4), we find a positive constant $C$ independent of $x, R$ and $t$ such that

$$
\frac{s}{C R^{\alpha}} \geq 1 \text { on } \partial B(x, 3 R / 2)
$$

Let $u$ be the capacitary potential for $\bar{B}(x, t R)$ in $B(x, 2 R)$, i.e.,

$$
\begin{aligned}
& \Delta u=0 \text { in } B(x, 2 R) \backslash \bar{B}(x, t R), \\
& u=1 \text { on } \bar{B}(x, t R), \\
& u=0 \text { on } \partial B(x, 2 R), \\
& \operatorname{Cap}_{B(x, 2 R)}(\bar{B}(x, t R))=\int_{B(x, 2 R)}|\nabla u|^{2} d \mu .
\end{aligned}
$$


Since $1-u \leq s /\left(C R^{\alpha}\right)$ on $\partial B(x, 3 R / 2)$, it follows from the maximum principle

$$
1-u \leq \frac{s}{C R^{\alpha}} \approx \frac{\operatorname{dist}\left(\cdot, E_{t}\right)^{\alpha}}{R^{\alpha}} \text { in } B(x, 3 R / 2) \backslash \bar{B}(x, t R) .
$$

Hence

$$
u \geq 1-C \frac{((1-t) R)^{\alpha}}{R^{\alpha}}=1-C(1-t)^{\alpha} \quad \text { in } B(x, R) \backslash \bar{B}(x, t R)
$$

with another positive constant $C$. If $1-C(1-t)^{\alpha}>0$, then by definition,

$$
\begin{aligned}
\operatorname{Cap}_{B(x, 2 R)}(\bar{B}(x, R)) & \leq \frac{1}{\left(1-C(1-t)^{\alpha}\right)^{2}} \int_{B(x, 2 R)}|\nabla u|^{2} d \mu \\
& =\frac{\operatorname{Cap}_{B(x, 2 R)}(\bar{B}(x, t R))}{\left(1-C(1-t)^{\alpha}\right)^{2}} .
\end{aligned}
$$

Hence

$$
\frac{\operatorname{Cap}_{B(x, 2 R)}(\bar{B}(x, t R))}{\operatorname{Cap}_{B(x, 2 R)}(\bar{B}(x, R))} \geq\left(1-C(1-t)^{\alpha}\right)^{2}
$$

so that the lemma follows as $\lim _{t \rightarrow 1}\left(1-C(1-t)^{\alpha}\right)^{2}=1$.

Proof of Theorem 1.3 By definition the first inequality holds for arbitrary open sets $D$. Let us prove the second inequality. In view of Lemma 5.3, we find an integer $N \geq 2$ depending only on $\sqrt{K} R_{0}$ and $n$ such that

$$
\frac{\operatorname{Cap}_{B(x, 2 R)}\left(\bar{B}\left(x,\left(1-N^{-1}\right) R\right)\right)}{\operatorname{Cap}_{B(x, 2 R)}(\bar{B}(x, R))} \geq \sqrt{\eta}
$$

uniformly for $x \in M$ and $0<R<R_{0}$. Let $C_{5}$ be as in Lemma 4.6 and take an integer $k>2$ so large that $\left(1-C_{5}^{-1} \eta^{\prime}\right)^{k} \leq 1-\sqrt{\eta}$.

Let $w_{\eta}(D)<R_{0}$. We prove the theorem by showing

$$
w_{\eta}(D) \leq 2 N k w_{\eta^{\prime}}(D)
$$

If $w_{\eta^{\prime}}(D) \geq R_{0} /(2 N k)$, then $w_{\eta}(D)<R_{0} \leq 2 N k w_{\eta^{\prime}}(D)$, so (5.6) follows. Suppose

$$
w_{\eta^{\prime}}(D)<\frac{R_{0}}{2 N k}
$$

For simplicity we write $\rho=w_{\eta^{\prime}}(D)$. Apply Lemma 4.7, with $\eta^{\prime}$ in place of $\eta$, to $x \in D$ and $R=2 N k \rho$. We obtain

$$
\sup _{D \cap \bar{B}(x, R-2 k \rho)} \omega(D \cap \partial B(x, R), D \cap B(x, R)) \leq\left(1-C_{5}^{-1} \eta^{\prime}\right)^{k} \leq 1-\sqrt{\eta} .
$$


Let $E=\bar{B}(x, R) \backslash D$. Then the maximum principle yields $\omega(\partial B(x, 2 R), B(x, 2 R) \backslash E) \leq \omega(D \cap \partial B(x, R), D \cap B(x, R))$ in $D \cap B(x, R)$, so that

$$
\omega(\partial B(x, 2 R), B(x, 2 R) \backslash E) \leq 1-\sqrt{\eta} \text { in } \bar{B}(x, R-2 k \rho),
$$

where we use the convention $\omega(\partial B(x, 2 R), B(x, 2 R) \backslash E)=0$ in $E$. Hence, Lemma 4.6 (i) with $R-2 k \rho$ and $2 R$ in place of $r$ and $R$ gives

$$
1-\frac{\operatorname{Cap}_{B(x, 2 R)}(E)}{\operatorname{Cap}_{B(x, 2 R)}(\bar{B}(x, R-2 k \rho))} \leq 1-\sqrt{\eta},
$$

so that

$$
\frac{\operatorname{Cap}_{B(x, 2 R)}(E)}{\operatorname{Cap}_{B(x, 2 R)}(\bar{B}(x, R-2 k \rho))} \geq \sqrt{\eta} .
$$

Multiplying the inequality and (5.5), we obtain

$$
\frac{\operatorname{Cap}_{B(x, 2 R)}(E)}{\operatorname{Cap}_{B(x, 2 R)}(\bar{B}(x, R))} \geq \eta,
$$

as $R-2 k \rho=\left(1-N^{-1}\right) R$. Since $x \in D$ is arbitrary, we have $w_{\eta}(D)<R=2 N k \rho=$ $2 N k w_{\eta^{\prime}}(D)$. Thus we have (5.6).

Proof of Theorem 5.1 First, let us prove the second inequality of (5.1), i.e., $\left\|v_{D}\right\|_{\infty} \leq$ $C w_{\eta}(D)^{2}$. In view of the monotonicity of the torsion function, we may assume that $D$ is bounded and hence $\left\|v_{D}\right\|_{\infty}<\infty$. By definition we find $r, w_{\eta}(D) \leq r<2 w_{\eta}(D)<$ $2 R_{0}$, such that

$$
\frac{\operatorname{Cap}_{B(x, 2 r)}(\bar{B}(x, r) \backslash D)}{\operatorname{Cap}_{B(x, 2 r)}(\bar{B}(x, r))} \geq \eta \quad \text { for every } x \in D
$$

For a moment we fix $x \in D$ and let $B=B(x, r), B^{*}=B(x, 2 r)$, and $E=\bar{B} \backslash D$ for simplicity. Then $\operatorname{Cap}_{B^{*}}(E) / \operatorname{Cap}_{B^{*}}(\bar{B}) \geq \eta$. We compare $v_{D}$ with

$$
v_{B^{*}}=\int_{B^{*}} G_{B^{*}}(\cdot, y) d \mu(y) .
$$

It is easy to see that $v_{D}-v_{B^{*}}$ is harmonic in $D \cap B^{*}$ and $v_{D}=0$ on $\partial D$ outside a polar set. Hence the maximum principle yields

$$
v_{D}-v_{B^{*}} \leq\left\|v_{D}\right\|_{\infty} \omega\left(D \cap \partial B^{*}, D \cap B^{*}\right) \text { in } D \cap B^{*}
$$


Since Lemma 4.6 implies that

$$
\omega^{x}\left(D \cap \partial B^{*}, D \cap B^{*}\right) \leq \omega^{x}\left(\partial B^{*}, B^{*} \backslash E\right) \leq 1-C_{5}^{-1} \eta,
$$

it follows from Lemma 4.2 that

$$
v_{D}(x) \leq v_{B^{*}}(x)+\left\|v_{D}\right\|_{\infty} \omega^{x}\left(D \cap \partial B^{*}, D \cap B^{*}\right) \leq C r^{2}+\left\|v_{D}\right\|_{\infty}\left(1-C_{5}^{-1} \eta\right)
$$

Taking the supremum with respect to $x \in D$, we obtain

$$
\left\|v_{D}\right\|_{\infty} \leq C C_{5} \eta^{-1} r^{2} \leq 4 C C_{5} \eta^{-1} w_{\eta}(D)^{2} .
$$

Second, let us prove the first inequality of (5.1), i.e. $w_{\eta}(D)^{2} \leq C\left\|v_{D}\right\|_{\infty}$. We distinguish two cases. Suppose first $\left\|v_{D}\right\|_{\infty} \geq C_{3} R_{0}^{2} / 2$ with $C_{3}$ as in (4.1). Then

$$
\left\|v_{D}\right\|_{\infty} \geq \frac{1}{2} C_{3} R_{0}^{2}>\frac{1}{2} C_{3} w_{\eta}(D)^{2}
$$

as required. Suppose next $\left\|v_{D}\right\|_{\infty}<C_{3} R_{0}^{2} / 2$. Take $R$ such that

$$
\left\|v_{D}\right\|_{\infty}=\frac{C_{3} R^{2}}{2}
$$

Then $0<R<R_{0}$. Let $x \in D$. This time, we let $B=B(x, R), B^{*}=B(x, 2 R)$ and $E=\bar{B} \backslash D$ with $R$ as in (5.7). We shall compare $v_{D}$ with the torsion function

$$
v_{B}=\int_{B} G_{B}(\cdot, y) d \mu(y) .
$$

Observe that $v_{B}-v_{D}$ is harmonic in $D \cap B$. By the maximum principle and Lemma 4.2

$$
\begin{aligned}
v_{B}-v_{D} & \leq \sup _{E} v_{B} \cdot \omega(\partial E, B \backslash E)=\sup _{E} v_{B} \cdot(1-\omega(D \cap \partial B, B \backslash E)) \\
& \leq C R^{2}\left(1-\omega\left(\partial B^{*}, B^{*} \backslash E\right)\right) \text { in } D \cap B,
\end{aligned}
$$

since $\partial(D \cap B) \subset(B \cap \partial D) \cup(D \cap \partial B) \subset E \cup \partial B$, and since $v_{B}=0$ on $\partial B$. Let $0<\varepsilon<1$ be as in (4.1). Taking the infimum over $\bar{B}(x, \varepsilon R)$, we obtain from Lemma 4.6 that

$$
\begin{aligned}
\inf _{\bar{B}(x, \varepsilon R)} v_{B}-\left\|v_{D}\right\|_{\infty} & \leq C R^{2}\left(1-\sup _{\bar{B}(x, \varepsilon R)} \omega\left(\partial B^{*}, B^{*} \backslash E\right)\right) \\
& \leq C R^{2} \frac{\operatorname{Cap}_{B^{*}}(E)}{\operatorname{Cap}_{B^{*}}(\bar{B}(x, \varepsilon R))}
\end{aligned}
$$


Hence, (4.1) and (5.7) yield

$$
C_{3} R^{2}-\frac{C_{3} R^{2}}{2} \leq C R^{2} \frac{\operatorname{Cap}_{B^{*}}(E)}{\operatorname{Cap}_{B^{*}}(\bar{B}(x, \varepsilon R))} .
$$

Dividing by $C R^{2}$, we obtain

$$
\frac{\operatorname{Cap}_{B^{*}}(E)}{\operatorname{Cap}_{B^{*}}(\bar{B}(x, \varepsilon R))} \geq \frac{C_{3}}{2 C},
$$

so that, by Lemma 4.4 and volume doubling

$$
\begin{aligned}
\frac{\operatorname{Cap}_{B^{*}}(E)}{\operatorname{Cap}_{B^{*}}(\bar{B}(x, R))} & =\frac{\operatorname{Cap}_{B^{*}}(E)}{\operatorname{Cap}_{B^{*}}(\bar{B}(x, \varepsilon R))} \cdot \frac{\operatorname{Cap}_{B^{*}}(\bar{B}(x, \varepsilon R))}{\operatorname{Cap}_{B^{*}}(\bar{B}(x, R))} \\
& \geq \frac{C_{3}}{2 C} \cdot \frac{C \mu(\bar{B}(x, \varepsilon R))}{\mu(\bar{B}(x, R))} \geq \eta^{\prime}
\end{aligned}
$$

with $0<\eta^{\prime}<1$ depending only on $\sqrt{K} R_{0}$ and $n$. Thus

$$
\frac{\operatorname{Cap}_{B^{*}}(\bar{B}(x, R) \backslash D)}{\operatorname{Cap}_{B^{*}}(\bar{B}(x, R))} \geq \eta^{\prime} .
$$

Since $x \in D$ is arbitrary, we have $w_{\eta^{\prime}}(D)<R$ and so $w_{\eta}(D) \leq C R$ by Theorem 1.3. Hence $w_{\eta}(D)^{2} \leq C\left\|v_{D}\right\|_{\infty}$ by (5.7). The proof is complete.

\section{Proof of Theorem 1.5}

The crucial step of the proof of Theorem 1.5 is the following parabolic box argument (cf. [1, Lem. 4.1]),

Lemma 6.1 Suppose (1.2) holds. If $t>0$, then

$$
P_{D}(t, x) \leq C_{t} G_{D}(x, o) \text { for } x \in D
$$

with $C_{t}$ depending on $t$.

Proof Without loss of generality we may assume that $\tau=1$ in (1.2). For notational convenience we shall prove (6.1) with $T$ in place of $t$. For simplicity we write $w_{\eta}\left(G_{D}^{o}<\right.$ $s)=w_{\eta}\left(\left\{x \in D: G_{D}(x, o)<s\right\}\right.$. Let $\alpha_{j}=\exp \left(-2^{j}\right)$. Since

$$
\begin{aligned}
\int_{\alpha_{j}}^{\alpha_{j-1}} w_{\eta}\left(G_{D}^{o}<s\right)^{2} \frac{d s}{s} & \geq w_{\eta}\left(G_{D}^{o}<\alpha_{j}\right)^{2} \int_{\alpha_{j}}^{\alpha_{j-1}} \frac{d s}{s} \\
& =w_{\eta}\left(G_{D}^{o}<\alpha_{j}\right)^{2}\left(2^{j}-2^{j-1}\right)=2^{j-1} w_{\eta}\left(G_{D}^{o}<\alpha_{j}\right)^{2},
\end{aligned}
$$




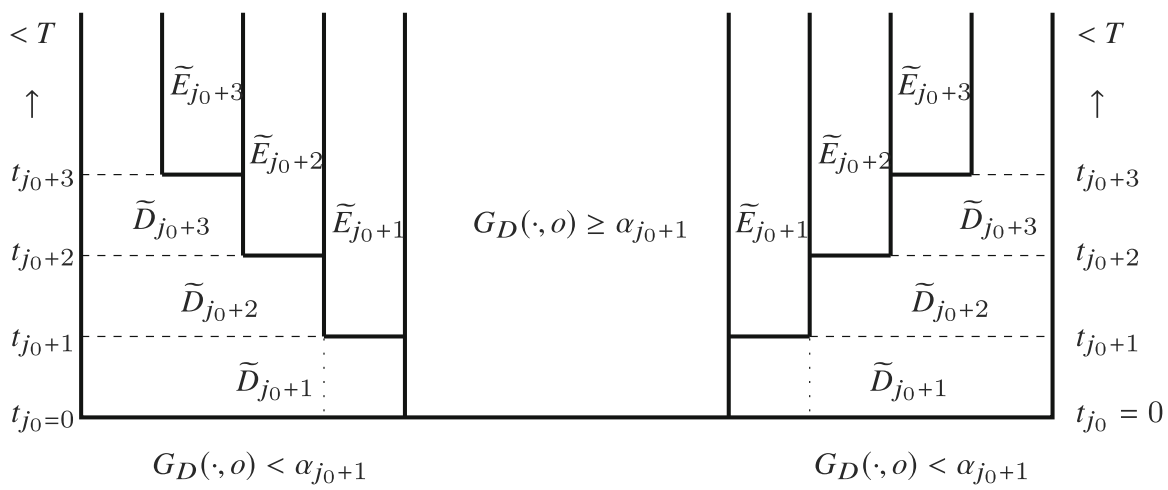

Fig. 1 Parabolic box argument

it follows from (1.2) that $\sum_{j=0}^{\infty} 2^{j} w_{\eta}\left(G_{D}^{o}<\alpha_{j}\right)^{2}<\infty$.

Let $w_{\eta}\left(G_{D}^{o}<1\right)<R_{0}<\infty$ and choose $C_{6}$ and $C_{7}$ as in Theorem 5.2. We find $j_{0} \geq 0$ such that

$$
\frac{3}{C_{7}} \sum_{j=j_{0}+1}^{\infty} 2^{j} w_{\eta}\left(G_{D}^{o}<\alpha_{j}\right)^{2}<T
$$

Define

$$
t_{k}=\frac{3}{C_{7}} \sum_{j=j_{0}+1}^{k} 2^{j} w_{\eta}\left(G_{D}^{o}<\alpha_{j}\right)^{2} \quad \text { for } k \geq j_{0}+1
$$

and $t_{j_{0}}=0$. Then $t_{k}$ increases and $\lim _{k \rightarrow \infty} t_{k}<T$ by (6.2). Observe that

$$
\frac{1}{\alpha_{k+1}} \exp \left(-\frac{C_{7}\left(t_{k}-t_{k-1}\right)}{w_{\eta}\left(G_{D}^{o}<\alpha_{k}\right)^{2}}\right)=\exp \left(2^{k+1}-3 \cdot 2^{k}\right)=\exp \left(-2^{k}\right)
$$

for $k \geq j_{0}+1$.

Let $D_{k}=\left\{x \in D: G_{D}(x, o)<\alpha_{k}\right\}, E_{k}=\left\{x \in D: \alpha_{k+1} \leq G_{D}(x, o)<\alpha_{k}\right\}$, $\widetilde{D}_{k}=\left(t_{k-1}, \infty\right) \times D_{k}$ and $\widetilde{E}_{k}=\left(t_{k}, \infty\right) \times E_{k}$. Put

$$
q_{k}=\sup _{(t, x) \in \widetilde{E}_{k}} \frac{P_{D}(t, x)}{G_{D}(x, o)}
$$

We claim that $\sup _{k \geq j_{0}+1} q_{k} \leq C$, which implies (6.1) with $T$ in place of $t$, and $C_{T}=\max \left\{C, 1 / \alpha_{j_{0}+1}\right\}$ since $(T, \infty) \times\left\{x \in D: G_{D}(x, o)<\alpha_{j_{0}+1}\right\} \subset \cup_{k \geq j_{0}+1} \widetilde{E}_{k}$ by (6.2). See Fig. 1 .

By the parabolic comparison principle over $\widetilde{D}_{j_{0}+1}$ we have

$$
P_{D}(t, x) \leq \frac{G_{D}(x, o)}{\alpha_{j_{0}+1}}+P_{D_{j_{0}+1}}(t, x) \text { for }(t, x) \in \widetilde{D}_{j_{0}+1}=(0, \infty) \times D_{j_{0}+1}
$$


Divide both sides by $G_{D}(x, o)$ and take the supremum over $\widetilde{E}_{j_{0}+1}$. Then (5.2) and (6.3) yield

$$
\begin{aligned}
q_{j_{0}+1} & \leq \frac{1}{\alpha_{j_{0}+1}}+\sup _{(t, x) \in \widetilde{E}_{j_{0}+1}} \frac{P_{D_{j_{0}+1}}(t, x)}{G_{D}(x, o)} \\
& \leq \frac{1}{\alpha_{j_{0}+1}}+\frac{C_{6}}{\alpha_{j_{0}+2}} \sup _{t \geq t_{j_{0}+1}} \exp \left(-\frac{C_{7} t}{w_{\eta}\left(D_{j_{0}+1}\right)^{2}}\right) \\
& \leq \frac{1}{\alpha_{j_{0}+1}}+\frac{C_{6}}{\alpha_{j_{0}+2}} \exp \left(-\frac{C_{7}\left(t_{j_{0}+1}-t_{j_{0}+1}\right)}{w_{\eta}\left(D_{j_{0}+1}\right)^{2}}\right)=\exp \left(2^{j_{0}+1}\right)+C_{6} \exp \left(-2^{j_{0}+1}\right)
\end{aligned}
$$

Let $k \geq j_{0}+2$. By the parabolic comparison principle over $\widetilde{D}_{k}$ we have

$$
P_{D}(t, x) \leq q_{k-1} G_{D}(x, o)+P_{D_{k}}\left(t-t_{k-1}, x\right) \text { for }(t, x) \in \widetilde{D}_{k}=\left(t_{k-1}, \infty\right) \times D_{k}
$$

Divide both sides by $G_{D}(x, o)$ and take the supremum over $\widetilde{E}_{k}$. In the same way as above, we obtain from (5.2) and (6.3) that

$$
q_{k} \leq q_{k-1}+\frac{C_{6}}{\alpha_{k+1}} \exp \left(-\frac{C_{7}\left(t_{k}-t_{k-1}\right)}{w_{\eta}\left(D_{k}\right)^{2}}\right) \leq q_{k-1}+C_{6} \exp \left(-2^{k}\right)
$$

Hence we have the claim as

$$
\sup _{k \geq j_{0}+1} q_{k} \leq \exp \left(2^{j_{0}+1}\right)+C_{6} \sum_{k=j_{0}+1}^{\infty} \exp \left(-2^{k}\right)<\infty .
$$

The lemma is proved.

Proof of Theorem 1.5 By Theorem 1.4 we have the first condition for IU. Let us show (1.1) for every $t>0$. It is known that the lower estimate of (1.1) follows from the upper estimate. Moreover, if $p_{D}\left(t_{0}, x, y\right) \leq C_{t_{0}} \varphi_{D}(x) \varphi_{D}(y)$ for all $x, y \in D$ with some $t_{0}>0$, then $p_{D}(t, x, y) \leq C_{t} \varphi_{D}(x) \varphi_{D}(y)$ holds with $C_{t} \leq C_{t_{0}} e^{-\lambda_{D}\left(t-t_{0}\right)}$ for $t \geq t_{0}$ (See e.g. [1, Prop. 2.1]). Hence, it suffices to show the upper estimate of (1.1) for small $t>0$.

Since $\varphi_{D}$ is superharmonic, and since $G_{D}(\cdot, o)$ is harmonic outside $\{o\}$, we have $G_{D}(\cdot, o) \leq C \varphi_{D}$ apart from a neighborhood of $o$. So, it is sufficient to show that if $t>0$ small, then there exists $C_{t}>0$ such that

$$
p_{D}(t, x, y) \leq C_{t} G_{D}(x, o) G_{D}(y, o) \text { for } x, y \in D \text {. }
$$

Let $i_{0}$ be the injectivity radius of $M$. It is known that

$$
\mu(B(x, r)) \geq C r^{n} \text { for } 0<r<i_{0} / 2 \text { and } x \in M \text {. }
$$


where $C>0$ depends only on $M$ (Croke [9, Prop. 14]). Hence, the Gaussian estimate (2.1) yields

$$
p_{M}(t, x, y) \leq \frac{C}{V(x, \sqrt{t})} \leq C t^{-n / 2}
$$

for $0<t<\min \left\{R_{0}^{2},\left(i_{0} / 2\right)^{2}\right\}$ and $x, y \in M$. Let $0<t<\min \left\{R_{0}^{2},\left(i_{0} / 2\right)^{2}\right\}$ and $x, y, z \in D$. By (6.5) we have

$$
\begin{aligned}
p_{D}(2 t, z, y) & =\int_{D} p_{D}(t, z, w) p_{D}(t, w, y) d \mu(w) \\
& \leq \int_{D} p_{M}(t, z, w) p_{D}(t, w, y) d \mu(w) \\
& \leq C t^{-n / 2} \int_{D} p_{D}(t, w, y) d \mu(w)=C t^{-n / 2} P_{D}(t, y),
\end{aligned}
$$

since the heat kernel is symmetric. Moreover,

$$
\begin{aligned}
p_{D}(3 t, x, y) & \leq \int_{D} p_{D}(t, x, z) p_{D}(2 t, z, y) d \mu(z) \\
& \leq \int_{D} p_{D}(t, x, z) C t^{-n / 2} P_{D}(t, y) d \mu(z) \\
& =C t^{-n / 2} P_{D}(t, x) P_{D}(t, y) .
\end{aligned}
$$

Hence Lemma 6.1 yields

$$
p_{D}(3 t, x, y) \leq C t^{-n / 2} P_{D}(t, x) P_{D}(t, y) \leq C_{t} G_{D}(x, o) G_{D}(y, o) .
$$

Replacing $3 t$ by $t$, we obtain (6.4) for small $t>0$. Thus the theorem is proved.

Remark 6.2 The assumption on the injectivity radius can be replaced by

$$
\inf _{x \in M} \mu\left(B\left(x, R_{0}\right)\right)>0 .
$$

In fact, (2.2) yields

$$
\mu(B(x, r)) \geq C\left(\frac{r}{R_{0}}\right)^{\alpha} \inf _{x \in M} \mu\left(B\left(x, R_{0}\right)\right) \text { for all } x \in M \text { and } 0<r<R_{0},
$$

and hence for small $t>0$,

$$
p_{M}(t, x, y) \leq \frac{C}{V(x, \sqrt{t})} \leq C t^{-\alpha / 2}
$$

Replacing (6.5) by this inequality, we obtain

$$
p_{D}(3 t, x, y) \leq C t^{-\alpha / 2} P_{D}(t, x) P_{D}(t, y) \leq C_{t} G_{D}(x, o) G_{D}(y, o),
$$


which proves Theorem 1.5. See [10] for further discussion on (6.6).

\section{Remarks}

Once we obtain the theorems in Sect. 1, we can extend many Euclidean results to the setting of manifolds. Proofs are almost the same as in the Euclidean case. For instance, we relax the requirement of inner uniformity for IU assumed in [15, Thm. 7.9]. For a curve $\gamma$ in $M$ we denote the length of $\gamma$ and the subarc of $\gamma$ between $x$ and $y$ by $\ell(\gamma)$ and $\gamma(x, y)$, respectively. For a domain $D$ in $M$ we define the inner metric in $D$ as

$$
d_{D}(x, y)=\inf \{\ell(\gamma): \gamma \text { is a curve connecting } x \text { and } y \text { in } D\}
$$

Definition 7.1 Let $D$ be a domain in $M$ and let $\delta_{D}(x)=\operatorname{dist}(x, M \backslash D)$.

(i) We say that $D$ is a John domain if there exist $o \in D$ and $C \geq 1$ such that every $x \in D$ is connected to $o$ by a rectifiable curve $\gamma \subset D$ with the property

$$
\ell(\gamma(x, z)) \leq C \delta_{D}(z) \text { for all } z \in \gamma
$$

(ii) We say that $D$ is an inner uniform domain if there exists $C \geq 1$ such that every pair of points $x, y \in D$ can be connected by a rectifiable curve $\gamma \subset D$ with the properties $\ell(\gamma) \leq C d_{D}(x, y)$ and

$$
\min \left\{\ell \left(\gamma(x, z), \ell(\gamma(z, y)\} \leq C \delta_{D}(z) \text { for all } z \in \gamma\right.\right.
$$

If we replace $d_{D}(x, y)$ by the ordinary metric $d(x, y)$ in (ii), then we obtain a uniform domain. By definition a John domain is necessarily bounded. We have the following inclusions for these classes of bounded domains:

$$
\text { uniform } \varsubsetneqq \text { inner uniform } \varsubsetneqq \text { John. }
$$

Figure 2 depicts a John domain that is not inner uniform. We find a curve connecting $x$ and $o$ with the property of Definition 7.1 (i); yet there is no curve connecting $x$ and

Fig. 2 A John domain that is not inner uniform

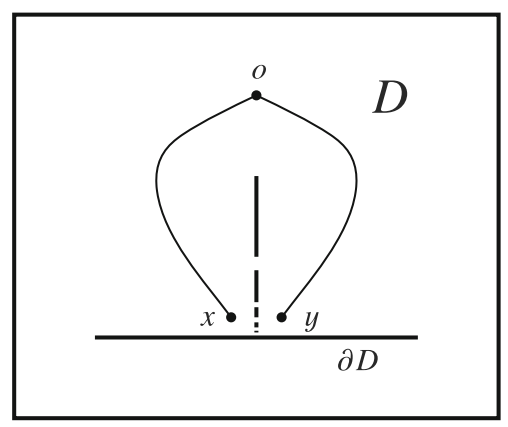


$y$ with the properties of Definition 7.1 (ii) if the gaps on the vertical segment shrink sufficiently fast.

\section{Theorem 7.2 A John domain is IU.}

Proof Let $D$ be a John domain. Observe that $w_{\eta}\left(\left\{x \in D: \delta_{D}(x)<r\right\}\right) \leq C r$ for small $r>0$ by definition and $G_{D}(x, o) \geq C \delta_{D}(x)^{\alpha}$ with some $\alpha>0$ by the Harnack inequality. Hence

$$
w_{\eta}\left(\left\{x \in D: G_{D}(x, o)<t\right\}\right) \leq w_{\eta}\left(\left\{x \in D: \delta_{D}(x)<(t / C)^{1 / \alpha}\right\}\right) \leq C t^{1 / \alpha},
$$

so that (1.2) holds. Therefore Theorem 1.5 asserts that $D$ is IU.

Acknowledgements The authors would like to thank the referee for his/her careful reading of the manuscript and many useful suggestions.

Open Access This article is licensed under a Creative Commons Attribution 4.0 International License, which permits use, sharing, adaptation, distribution and reproduction in any medium or format, as long as you give appropriate credit to the original author(s) and the source, provide a link to the Creative Commons licence, and indicate if changes were made. The images or other third party material in this article are included in the article's Creative Commons licence, unless indicated otherwise in a credit line to the material. If material is not included in the article's Creative Commons licence and your intended use is not permitted by statutory regulation or exceeds the permitted use, you will need to obtain permission directly from the copyright holder. To view a copy of this licence, visit http://creativecommons.org/licenses/by/4.0/.

\section{References}

1. Aikawa, H.: Intrinsic ultracontractivity via capacitary width. Rev. Mat. Iberoam. 31(3), 1041-1106 (2015)

2. Ancona, A.: On strong barriers and an inequality of Hardy for domains in $\mathbf{R}^{n}$. J. Lond. Math. Soc. (2) 34(2), 274-290 (1986)

3. Armitage, D.H., Gardiner, S.J.: Classical Potential Theory. Springer Monographs in Mathematics. London Ltd., London (2001)

4. Bañuelos, R.: Intrinsic ultracontractivity and eigenfunction estimates for Schrödinger operators. J. Funct. Anal. 100(1), 181-206 (1991)

5. Bañuelos, R., Davis, B.: A geometrical characterization of intrinsic ultracontractivity for planar domains with boundaries given by the graphs of functions. Indiana Univ. Math. J. 41(4), 885-913 (1992)

6. Barlow, M.T., Grigor'yan, A., Kumagai, T.: On the equivalence of parabolic Harnack inequalities and heat kernel estimates. J. Math. Soc. Jpn. 64(4), 1091-1146 (2012)

7. Bass, R.F., Burdzy, K.: Lifetimes of conditioned diffusions. Probab. Theory Related Fields 91(3-4), 405-443 (1992)

8. Cipriani, F.: Intrinsic ultracontractivity of Dirichlet Laplacians in nonsmooth domains. Potential Anal. 3(2), 203-218 (1994)

9. Croke, C.B.: Some isoperimetric inequalities and eigenvalue estimates. Ann. Sci. École Norm. Sup. (4) 13(4), 419-435 (1980)

10. Croke, C.B., Karcher, H.: Volumes of small balls on open manifolds: lower bounds and examples. Trans. Amer. Math. Soc. 309(2), 753-762 (1988)

11. Davies, E.B.: Heat Kernels and Spectral Theory. Cambridge Tracts in Mathematics, vol. 92. Cambridge University Press, Cambridge (1989)

12. Davies, E.B., Simon, B.: Ultracontractivity and the heat kernel for Schrödinger operators and Dirichlet Laplacians. J. Funct. Anal. 59(2), 335-395 (1984)

13. Davis, B.: Intrinsic ultracontractivity and the Dirichlet Laplacian. J. Funct. Anal. 100(1), 162-180 (1991) 
14. Grigor'yan, A.: Heat Kernel and Analysis on Manifolds. AMS/IP Studies in Advanced Mathematics, vol. 47. American Mathematical Society, International Press, Boston, MA, Providence (2009)

15. Lierl, J., Saloff-Coste, L.: The Dirichlet heat kernel in inner uniform domains: local results, compact domains and non-symmetric forms. J. Funct. Anal. 266(7), 4189-4235 (2014)

16. Méndez-Hernández, P.J.: Toward a geometric characterization of intrinsic ultracontractivity for Dirichlet Laplacians. Michigan Math. J. 47(1), 79-99 (2000)

17. Persson, A.: Bounds for the discrete part of the spectrum of a semi-bounded Schrödinger operator. Math. Scand. 8, 143-153 (1960)

18. Saloff-Coste, L.: Aspects of Sobolev-Type Inequalities. London Mathematical Society Lecture Note Series, vol. 289. Cambridge University Press, Cambridge (2002)

19. van den Berg, M.: Spectral bounds for the torsion function. Integral Equ. Oper. Theory 88(3), 387-400 (2017)

Publisher's Note Springer Nature remains neutral with regard to jurisdictional claims in published maps and institutional affiliations. 